This is the peer reviewed version of the following article: Pérez Tomàs, Amador; Lira Cantú, Mónica; Catalan, Gustau. Above-Bandgap Photovoltages in Antiferroelectrics. Advanced Materials, vol.28, issue 43 (2016), p. 9644-9647, which has been published in final form at

https://onlinelibrary.wiley.com/doi/full/10.1002/adma.201603176. This article may be used for noncommercial purposes in accordance with Wiley Terms and Conditions for Use of Self-Archived Versions.

\title{
Above-bandgap Photovoltages in Antiferroelectrics
}

Amador Pérez-Tomás ${ }^{1 *}$, Monica Lira-Cantúl ${ }^{1}$ and Gustau Catalan ${ }^{1,2 * *}$

${ }^{1}$ Catalan Institute of Nanoscience and Nanotechnology (ICN2), CSIC and The Barcelona Institute of Science and Technology, Campus UAB, Bellaterra, 08193 Barcelona, Spain

${ }^{2}$ Institut Català de Recerca i Estudis Avançats (ICREA), Barcelona 08010, Catalonia, Spain.

e-mail: * amador.perez@icn2.cat ;** gustau.catalan@icn2.cat

Keywords: (antiferroelectrics, photovoltaics, above bandgap voltages)

The ability of some materials to generate photovoltages greater than their bandgap has been stimulating scientists for many decades. ${ }^{[1-4]}$ In piezoelectrics and ferroelectrics, above-bandgap photovoltages are enabled by the intrinsic asymmetry of the lattice. ${ }^{[5]}$ Antiferroelectrics, by contrast, are generally centrosymmetric, but application of a suitably large external field can cause a transition from their antipolar ground state to a polar phase. Though removing the external field should in principle result in a return to the antipolar state and thus the loss of any above-bandgap photovoltage, switching under illumination results in the pinning of the polar state. Here we show that this allows antiferroelectric thin films to generate above-bandgap photovoltages in excess of one hundred volts and photovoltaic fields of several megavolts per centimetre, the largest ever measured for any material. 
A general rule in conventional (i.e., semiconductor junction-based) photovoltaics is that the solar cell photovoltage must be lower than the bandgap of the semiconductor. ${ }^{[6]}$ However, an anomalous photovoltaic effect, whereby photovoltaic devices exhibit larger photovoltages than the bandgap, has been observed in many semiconductor systems since $1946,{ }^{[1]}$ which coincidentally is the same year which Ohl patented the modern conventional solar cell. ${ }^{[7]}$ These photovoltages are normally due to the cumulative effect of connecting in tandem several individual internal junctions, such as grain boundaries, ${ }^{[8]}$ and the tandem principle has also been invoked as an explanation for above-bandgap photovoltages in multiferroic $\mathrm{BiFeO}_{3}$ with parallel-stacked domain walls. ${ }^{[4]}$ In a different fashion, materials with a non-centrosymmetric structure can also develop above-bandgap photovoltages even in the absence of tandem effects. This is called the bulk photovoltaic effect $(B P E)^{[2]}$ and is believed to be a property of the lattice non-centrosymmetry, ${ }^{[2],[5],[9]}$ although it is not easy to separate out the intrinsic role of crystal symmetry (shift current theory ${ }^{[2],[10]}$ ) from extrinsic contributions from chemical potential gradients, ${ }^{[8]}$ grain boundaries, ${ }^{[3]}$ or domain walls. ${ }^{[4]}$ The largest above-bandgap photovoltages have been reported for the sub-set of piezoelectrics formed by ferroelectrics (i.e. polar materials where the polarization is reversible) such as $\mathrm{LiNbO}_{3},{ }^{[2]} \mathrm{BaTiO}_{3}{ }^{[11]}$ and $\mathrm{BiFeO}_{3}{ }^{[6],[12]}$

A close relative of ferroelectricity is antiferroelectricity. Antiferroelectricity was postulated by Kittel in $1951^{[13]}$ and demonstrated in $\mathrm{PbZrO}_{3}$ (PZO) the same year by Shirane and coworkers. ${ }^{[14]}$ In an antiferroelectric material, adjacent ferroelectric dipoles are aligned in opposite orientation, so that the net polarization is zero. The archetypal material, $\mathrm{PbZrO}_{3}$, for example, is centrosymmetric in its ground state (space group Pbam), ${ }^{[15]-[18]}$ but when a sufficiently large external voltage is applied the antiparallel dipoles can be realigned into a polar, ferroelectric-like state. Antiferroelectrics are promising for applications such as high-energy capacitors, ${ }^{[19]}$ high-strain actuators, cooling devices or pulsed voltage/current suppliers. ${ }^{[20]}$ 
As compared with their ferroelectric counterparts, however, little is known about the photoelectric properties of antiferroelectrics ${ }^{[21],[22]}$, and in particular their photovoltages and photocurrents have not yet been measured. And yet, antiferroelectrics can potentially provide an optimum material family to explore bulk photovoltaic effect, because even though their ground state is non-polar, a polar state can be "switched on" without changing the composition, grain structure, electrodes, thickness, or light absorption. The present investigation does precisely that. We have characterized the closed circuit photocurrent and open circuit photovoltage of antiferroelectric thin films both in their ground (antipolar) state and in their polarized state. A sharp transition happens from near zero to large $(<100 \mathrm{~V})$ photovoltages as the polarization is switched on, consistent with the activation of the bulk photovoltaic effect. The photoelectric field (photovoltage divided by thickness) of the antiferroelectric films is $>5 \mathrm{MV} / \mathrm{cm}$, about an order of magnitude bigger than the previous benchmark. Additionally, and importantly from a practical point of view, we observe that, once the bulk photovoltaic effect has been switched on, it remains even in the absence of external bias (i.e., antiferroelectric films that are polarized under illumination do not return to their antipolar ground state), meaning that antiferroelectrics can replace ferroelectrics without loss of photovoltaic functionality.

The AFE layers in this study have been grown by a solution processing method (sol-gel synthesis followed by spin coating deposition) onto fluorine-doped tin oxide (FTO), a transparent conducting oxide with low sheet resistance and a higher resilience to hightemperature processing than indium tin oxide and a standard for solar cells such as organometal trihalide perovskites. ${ }^{[23]}$ The growth details are found in the methods section; here, we just point out that solution-processing fabrication is relatively simple and readily up-scalable for photovoltaic cell manufacturing. ${ }^{[24]}$ Short exposure time to high temperature during the solution process helps preserve the integrity of the transparent bottom electrode, thus allowing the thin film device depicted in Figure 1 to be fabricated in a vertical configuration, instead of the more 
usual lateral architecture. ${ }^{[4],[12],[25],[26]}$ This allows the entire area of the device to be illuminated from below without any shadow effects. The parallel-plate configuration also has the fundamental advantage that the small distance between electrodes translates the devices' photovoltage into very large photoelectric fields (photovoltage divided by thickness).

Light absorption (see Methods and supplementary information) confirmed that the PZO films are, as expected, wide-band gap semiconductors with a gap of 3.7-3.8 eV (the same as for bulk PZO) ${ }^{[27]}$ and thus highly absorbing in the near-ultraviolet range. The antipolar phase becomes $\operatorname{polar}\left(P_{s} \sim 40 \mu \mathrm{C} / \mathrm{cm}^{2}\right)$ above a critical forward coercive field $\left(E_{F} \sim 250 \mathrm{kV} / \mathrm{cm}\right)$ (see hysteresis loop Figure 1-e). Conversely, as the electric-field is decreased from maximum values to zero, the system returns to the antipolar phase at a backward switching field $\left(E_{B} \sim 75 \mathrm{kVcm}^{-1}\right)$. The same hysteresis takes place for negative voltages. The result is the characteristic double hysteresis loop of antiferroelectrics. To minimize leakage contributions the frequency of the measurement was $1 \mathrm{kHz}$ although much slower loops can be measured without any noticeable change down to frequencies as slow as $40 \mathrm{~Hz}$ (see supplementary information). Importantly also, although some films show some residual polarization at $0 \mathrm{~V}$ in the hysteresis loops (i.e., a slight "triple hysteresis effect"), films with no residual polarization could also be obtained, showing identical photoresponse (see supplementary material).

The interplay between polar state and photovoltaic response is shown in Figure 2-a and 2-b. On a virgin sample, there is no short-circuit photocurrent, consistent with the antipolar nature of the ground state. As an external bias voltage is applied, the current remains negligible until suddenly, at the coercive voltage, a spike is observed, corresponding to the transient displacement current caused by the onset of polarization. Further spikes can be observed as the voltage is ramped up, caused by the switching of residual domains (Barkhausen jumps). 
Suprisingly, as the voltage is then ramped back down, the illuminated antiferroelectric does not switch back to its antipolar state. Instead, it stays poled, and as a consequence it displays both a short-circuit photocurrent and an above-bandgap open-circuit photovoltage.

The remanence of the photo-assisted polar state has been observed in photorefractive storage devices $^{[21]}$, and is useful for photovoltaic cells such as ours, as it means that no external bias needs to be supplied to maintain the above-bandgap response. It is attributed to the drift and accumulation near the interface of photogeneraged charge, which generates a strong spacecharge field that pins the polarization and locks the AFE in its polar state. In highly resistive samples, the charge drift and interfacial build-up is slow, on the scale of several minutes. The time evolution of the photovoltage and the photoconductivity is shown in Figure 2-c. The photovoltaic development time is in the order of the dielectric relaxation time, $\tau=\varepsilon / \sigma^{[2]}$, where $\sigma$ is the low voltage conductivity, and $\varepsilon=\varepsilon_{r} \varepsilon_{0}$ is the dielectric constant, with $\varepsilon_{r} \sim 70-100$ as calculated from the slope of the saturated hysteresis loops.

Though the shift current density is in principle an intrinsic property of the induced polar state, ${ }^{[2]}$ the photovoltage is the result of dividing this intrinsic photocurrent by the conductivity, which is an extrinsic property that depends on the free carrier concentration and the quality of the sample. ${ }^{[28]}$ The consequence is that, when conductivity is low, the photovoltage can reach very high values indeed: Figure 3-a shows the photovoltaic response of a representative antiferroelectric device reaching open circuit photovoltages $V_{o c}=120 \pm 10 \mathrm{~V}$, about 30 times larger than the bandgap and consistent with the bulk photovoltaic origin of the effect (more such responses coming from various devices from different batches are shown in the supplementary materials in order to illustrate the reproducibility of the results). At such high voltages, the slope of the current vs voltage can be seen to change abruptly. The reason is that the photoelectric field (photovoltage divided by thickness) of these devices is so large that it 
approaches values close to the breakdown strength of the film and/or the intrinsic photovoltaic limit posed by the Micheron criterion, ${ }^{[29]} E_{P V} \leq P / \varepsilon=5-7 \mathrm{MVcm}^{-1}$, where $P$ is the polarization of the films $\left(P \approx 40 \mu \mathrm{Ccm}^{-2}\right)$. When this limit is reached, the conductivity must increase so as to ensure that the current goes to zero at a sustainable open-circuit voltage, resulting in the observed sudden change in slope.

The parallel-plate architecture of our photovoltaic cells also serves to translate the already large photovoltage into an enormous photoelectric field $E_{p v}$. The PZO layer in our devices is $200 \mathrm{~nm}$ thick (see methods section), so $120 \mathrm{~V}$ are equivalent to a photoelectric field $E_{p v}=6.0 \pm 0.3$ $\mathrm{MVcm}^{-1}$, which is not only of the order of the intrinsic Micheron limit but is also the largest photoelectric field reported for any material, ${ }^{[1],[30]}$ overcoming by more than an order of magnitude the landmark value of $\mathrm{LiNbO}_{3}$ single crystals $\left(\sim 0.1 \mathrm{MVcm}^{-1}\right)^{[1]}$ (Figure 3-b). Antiferroelectrics thus emerge as a family of switchable photovoltaic materials that are not only a useful testing ground for the physics of the bulk photovoltaic effect, but a competitive new entry to the state of the art in above-bandgaph photovoltaic devices. 


\section{Methods}

\section{Sol-Gel oxide synthesis}

The synthesis of the PZO was done as follows: $7.49 \mathrm{~g}$ of $\mathrm{Zr}$-isopropoxide $\left(\mathrm{Zr}\left[\mathrm{OCH}\left(\mathrm{CH}_{3}\right)_{2}\right]_{4}, 70\right.$ wt. \% in 1-propanol, Sigma), $5 \mathrm{ml}$ acetic acid and $10 \mathrm{ml}$ of n-propanol were mixed together with constant stirring in a glass beaker. 6.68 gr of $\mathrm{Pb}$ acetate $(10 \%$ excess $)$ [lead(II) acetate trihydrate, $\left.\mathrm{Pb}\left(\mathrm{CH}_{3} \mathrm{CO}_{2}\right)_{2} \cdot 3 \mathrm{H}_{2} \mathrm{O}(99.999 \%)\right]$ were added to the above solution. The reaction mixture was heated at constant temperature of $85^{\circ} \mathrm{C}$ using a silicon oil bath until the complete dissolution of the $\mathrm{Pb}$ precursor. After cooling down, acetic acid and distilled water were added until a $40 \mathrm{ml} 0.4 \mathrm{M}$ solution was obtained. The as-prepared solution was used without further modification for spin coating deposition.

\section{Antiferroelectric photovoltaic device fabrication}

The FTO-coated glass substrates of $1.1 \mathrm{~mm}$ thickness were bought from Solems, model ASAHI 100 by CVD (70-100 ohm resistance, thickness of FTO layer $800 \AA$ A.). Before use, the substrates cleaned with soap water, mili-Q water and ethanol (99.5\%) for $20 \mathrm{~min}$, and finally ozone/UV treated for 20 minutes. $80 \mu 1$ of the as-prepared PZO solution was spin-coated on top of the FTO at $4000 \mathrm{rpm}$ with an acceleration of $2000 \mathrm{rpm} / \mathrm{s}$ for $40 \mathrm{~s}$. The solution was dried in a hot plate for $5 \mathrm{~min}$ at $150{ }^{\circ} \mathrm{C}$ and annealed at $600-650{ }^{\circ} \mathrm{C}$ for $5-10 \mathrm{~min}$ in air. The anode metal is evaporated silver $(100 \mathrm{~nm})$. The thermal evaporator is an Univex 350G model from Oerlikon Leybold Vaccum inside a GS Globebox Systemtechnik GmbH globe box. Parallel plate devices were defined by means of shadow masks with diameters of $100 \mu \mathrm{m}, 2000 \mu \mathrm{m}$ and $400 \mu \mathrm{m}$. 


\section{Characterization}

X-ray powder diffraction (XRD) analyses between 5 and 120 degrees in a RIGAKU Rotaflex RU200 B instrument, using CuK $\alpha 1$ radiation.

The UV-visible analysis of solutions and thin films were made in a Shimadzu 1800 spectrophotometer. The optical band gap was derived by the Tauc's relation; $\alpha h v=A\left(h v-E_{g}\right)^{n}$. Where, $\alpha$ is the absorbance, A is a constant, $h v$ is photon energy, $E_{g}$ is the allowed energy gap and $n=1 / 2$ for allowed direct transitions. The optical bandgap value perfectly agrees with previous literature. First principles band-structure calculations for bulk PZO show that, in practice, PZO has a direct band gap at the $X$ point of around $\sim 3.8 \mathrm{eV}$ [27]. The valence-band

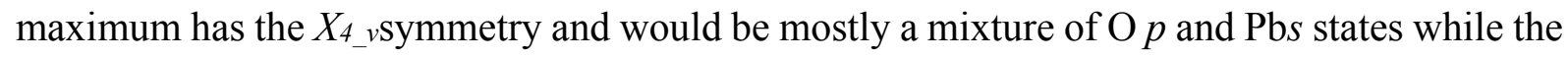
conduction-band minimum is an $X_{l c}$ state.

The UV-LED measurements were carried out in a home-made set-up attached to a probe station. The UV-LED source is a commercial UV LED from Roithner part APG2C1-365-E 1W $365 \mathrm{~nm}$. The AFE photovoltaic properties have been investigated by means of a high power UVA-light emitting diode. The LED is biased with $1 \mathrm{~W}$ DC power with a drive current of $350 \mathrm{~mA}$ and a voltage drop of around $3.5 \mathrm{~V}$. The nominal $365 \mathrm{~nm}$ UVA-LED irradiance has been precisely determined to be $I_{c}=159.1 \mathrm{~W} / \mathrm{m}^{2}$ with a spectrophotometer at the exact distance with the irradiation peak centered at $369.2 \mathrm{~nm}$ and a FWHM of $\Delta \lambda_{p}=9.6 \mathrm{~nm}$.

The I-V curves were measured with a Keithley model 6430 Sub-Femtoamp Remote SourceMeter.

The ferroelectric characterization was performed with a precision LC tester LCII Ferroelectric Test System unit of Radiant technologies attached to a probe station.

Spectral irradiance measurements were performed with a spectrophotometer SPECTRO 320 (D) release 5, 30932004, with gadget EOP-146 to measure the spectral irradiance. Spectral 
irradiance has been measured out-of-plane $\left(90^{\circ}\right)$ with a scan step of $1 \mathrm{~nm}$ and a speed of 100 $\mathrm{ms} / \mathrm{nm}$.

The TEM lamella was defined by a focused ion beam. The FIB has circuit editing capability and nanomachining capabilities. The FIB used in this work is a Zeiss 1560XB Cross Beam able to work with sample of size up to a $150 \mathrm{~mm}$. The field emission focused ion beam optics have a liquid Gallium ion emitter with acceleration voltage of $3 \mathrm{kV}$ to $30 \mathrm{kV}$ and beam current of $1 \mathrm{pA}-20 \mathrm{nA}$ with a maximum resolution of $7.0 \mathrm{~nm}$. The system contains a multiple injector needle system for 5 different gases including gas chemistry solution for ion or electron beam deposition of Platinum-containing material.

The TEM inspection has been carried out by a high-resolution FEI Tecnai F20 S/TEM equipped with an EDX. 


\section{Acknowledgements}

Authors acknowledge A. S. Amores for the lamella fabrication and Dr B. Ballesteros for the TEM inspection. To M. Tenorio for helping in the UV-Vis data extraction. Authors would like to thank N. Ribas, J. Cobo, J. Gallén, and X. Tomàs from WISMOK ESP Barcelona (Orbital 40) for their assistance in the UVA LED set-up. ICN2 acknowledges the support of the Spanish MINECO through the Severo Ochoa Centers of Excellence Program under Grant SEV-20130295, and the grants ENE2013-48816-C5-4-R and FIS2013-48668-C2-1-P. To the Agència de Gestió d'Ajuts Universitaris i de Recerca for the support to consolidated Catalonia research groups 2014SGR 1212 and 1216-F. To the COST Action StableNextSol project MP1307; and to the European Research Council (ERC) Starting Grant, project No. 308023. 


\section{References}

[1] J. Starkiewicz, L. Sosnowski, O. Simpson, Nature 1946, 158, 28.

[2] A. M. Glass, D. von der Linde, T. J. Negran, Appl. Phys. Lett. 1974, 25, 233-235.

[3] P. S. Brody, Journal of Solid State Chemistry, 1975, 12, 193-200.

[4] S. Y. Yang, J. Seidel, S. J. Byrnes, P. Shafer, C. H. Yang, M. D. Rossell, P. Yu, Y. H. Chu, J. F. Scott, J. W. Ager, Nat. Nanotechnol. 2010, 5, 143-147.

[5] P. J. Sturman, and V. M. Fridkin, Photovoltaic and Photo-refractive Effects in Noncentrosymmetric Materials, Gordon and Breach Science Publishers (1992).

[6] W. Shockley, H. J. Queisser, J. Appl. Phys. 1961, 32, 510.

[7] S. H. Ohl, Light-sensitive electric device, US2443542

[8] B. Goldstein, L. Pensak, J. Appl. Phys. 1959, 30, 155-161.

[9] W. Ruppel, R. Von Baltz, P. Würfel, Ferroelectrics 1982, 43, 109-123.

[10] S. M. Young, A. M. Rappe, Phys. Rev. Lett. 2012, 109, 116601.

[11]W. T. H Koch, R. Munser, W. Ruppel, P. Wurfel, Solid State Com. 1975, 17, 847-850.

[12]A. Bhatnagar, A. R. Chaudhuri, Y. H. Kim, D. Hesse, M. Alexe, Nat. Commun. 2013, 4, 2835.

[13]C. Kittel, Theory of antiferroelectric crystals, Phys. Rev. 1951, 82, 729-32.

[14] G. Shirane, E. Sawaguchi, Y. Takaui, Phys. Rev. 1951, 84, 476-81; E. Sawaguchi, H. Maniwa, S. Hoshino, Phys. Rev. 1951, 83, 1078.

[15]A. M. Glazer, K. Roleder, J. Dec, Acta Cryst. B 1993, 49, 846-52.

[16] S. Teslic, T. Egami, Acta Cryst. B 1998, 54, 750-765.

[17]H. Fujishita, Y. Ishikawa, S. Tanaka, A. Ogawaguchi, S. Katano, J. Phys. Soc. Jpn. 2003, $72,1426-1435$.

[18]J. Íñiguez, M. Stengel, S. Prosandeev, L. Bellaiche, Phys. Rev. B 2014, 90, 220103(R). 
[19] M. Ye, Q. Sun, X. Chen, Z. Jiang, F. Wang, J. Am. Ceram. Soc. 2011, 94, 3234-3236.

[20]X. Hao, J. Zhai, L. Bing Kong, Z. Xu, Progress in Materials Science 2014, 63, 1-57.

[21]C. E. Land, IEEE Trans. Electron. Dev. 1979, 26, 1143-1147.

[22]F. Wang, K. K. Li, G. H. Haertling, Appl. Phys. Lett. 1994, 65, 2507.

[23]M. M. Lee, J. Teuscher, T. Miyasaka, T. N. Murakami, H. J. Snaith, Science 2012, 338, 643-647.

[24] M. Graetzel, R. A. J. Janssen, D. B. Mitzi, E. H. Sargent, Nature 2012, 488, 304-312.

[25]K. Yao, B. K. Gan, M. Chen, S. Shannigrahi, Appl. Phys. Lett. 2005, 87, 212906.

[26] S. Nakashima, T. Uchida, D. Nakayama, H. Fujisawa, M. Kobune, M. Shimizu, J. J. Appl. Phys. 2014, 53(9S), 09PA16.

[27]H. Lee, Y. S. Kang, S-J. Cho, B. Xiao, H. Morkoç, T. Dong Kang, G. S. Lee, J. Li, S-H. Wei, P. G. Snyder, J. T. Evans, J. Appl. Phys. 2005, 98, 094108.

[28] V. I. Belinicher, B. I. Sturman, Soviet Physics Uspekhi 1980, 23, 199.

[29]F. Micheron, Ferroelectrics 1978, 21, 607-609.

[30]A. Zenkevich, Yu. Matveyev, K. Maksimova, R. Gaynutdinov, A. Tolstikhina, V. Fridkin, Phys. Rev. B 2014, 90, 161409(R). 


\section{Figure Captions}

Fig. 1 (a) Schematic view of the antiferroelectric above bandgap photovoltaic device comprising a single layer $(\sim 200 \mathrm{~nm})$ of lead zirconate $\left(\mathrm{PbZrO}_{3}\right.$ or $\left.\mathrm{PZO}\right)$ onto a transparent conductive oxide (FTO). (b) PZO has a perovskite crystal structure with a high temperature cubic structure and a room temperature distorted orthorhombic antiferroelectric phase. (c) and (d) photographs of the $\mathrm{PbZrO}_{3}$ films grown on glass with transparent bottom electrode. (e) Nested antiferroelectric double hysteresis loops, where the PZO exhibits a reversible transition from antipolar to polar under adequate voltage bias.

Fig. 2. (a) Antiferroelectric hysteresis loop measured in dark and (b) current $v s$ voltage response under illumination of an antiferroelectric thin film capacitor. The virgin antipolar phase shows no photocurrent, but when the film switches from the antipolar to the polar phase, a displacement current peak appears, after which a steady-state photocurrent is established and remains even when the external bias is switched off (short-circuit photocurrent). The induced polar state displays above-bandgap photovoltage. (c) time evolution of the photoconductivity and open-circuit photovoltage.

Fig. 3. (a) steady-state photovoltaic response of the capacitor, showing an open-circuit photovoltage in excess of $100 \mathrm{~V}$. (b) A timeline review (references are listed under Supplementary section III) of the reported photovoltaic fields for different above bangap and/or ferroelectric photovoltaic materials from the literature. Non-polar piezoelectrics $(\mathrm{CdTe}, \mathrm{ZnS}$, $\mathrm{PbS}$ ) stay below the $\sim \mathrm{kV} / \mathrm{cm}$ range, which is only achieved by ferroelectric materials such as $\mathrm{BTO}\left(\mathrm{BaTiO}_{3}\right), \mathrm{KNO}\left(\mathrm{KNbO}_{3}\right), \mathrm{LNO}\left(\mathrm{LiNbO}_{3}\right), \mathrm{BFO}\left(\mathrm{BiFeO}_{3}\right)$ and $\mathrm{BFCO}\left(\mathrm{Bi}_{2} \mathrm{FeCrO}_{6}\right)$. PVDF is polyvinylidene fluoride, a ferroelectric polymer. The antiferroelectric PZO photovoltaic cells are the first to reach the $\mathrm{MV} / \mathrm{cm}$ range. 


\section{Figures}

(a)
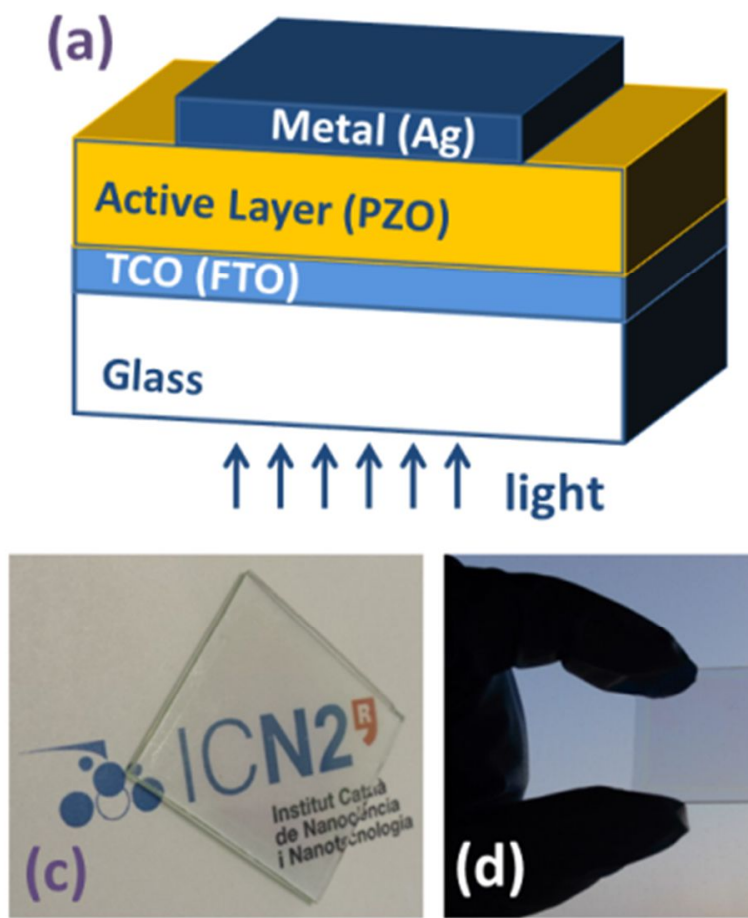

(b)

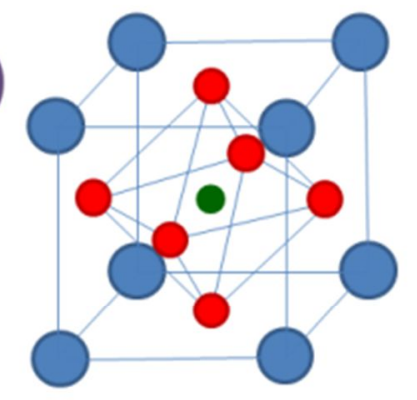

$\mathrm{Pb} \bigcirc \circ \bullet \mathrm{Zr}$

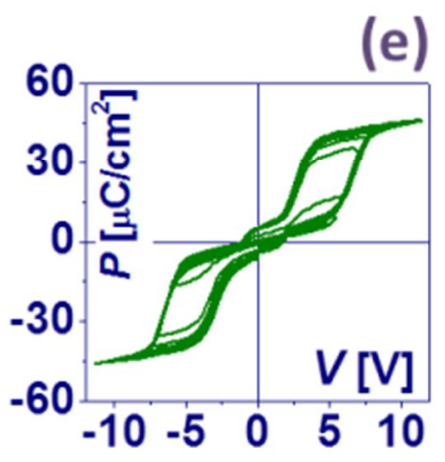

Fig. 1 (a) Schematic view of the antiferroelectric above bandgap photovoltaic device comprising a single layer $(200 \mathrm{~nm})$ of lead zirconate $\left(\mathrm{PbZrO}_{3}\right.$ or PZO) onto a transparent conductive oxide (FTO). (b) PZO has a perovskite crystal structure with a high temperature cubic structure and a room temperature distorted orthorhombic antiferroelectric phase. (c) and (d) photographs of the $\mathrm{PbZrO}_{3}$ films grown on glass with transparent bottom electrode. (e) Nested antiferroelectric double hysteresis loops, where the PZO exhibits a reversible transition from antipolar to polar under adequate voltage bias. 

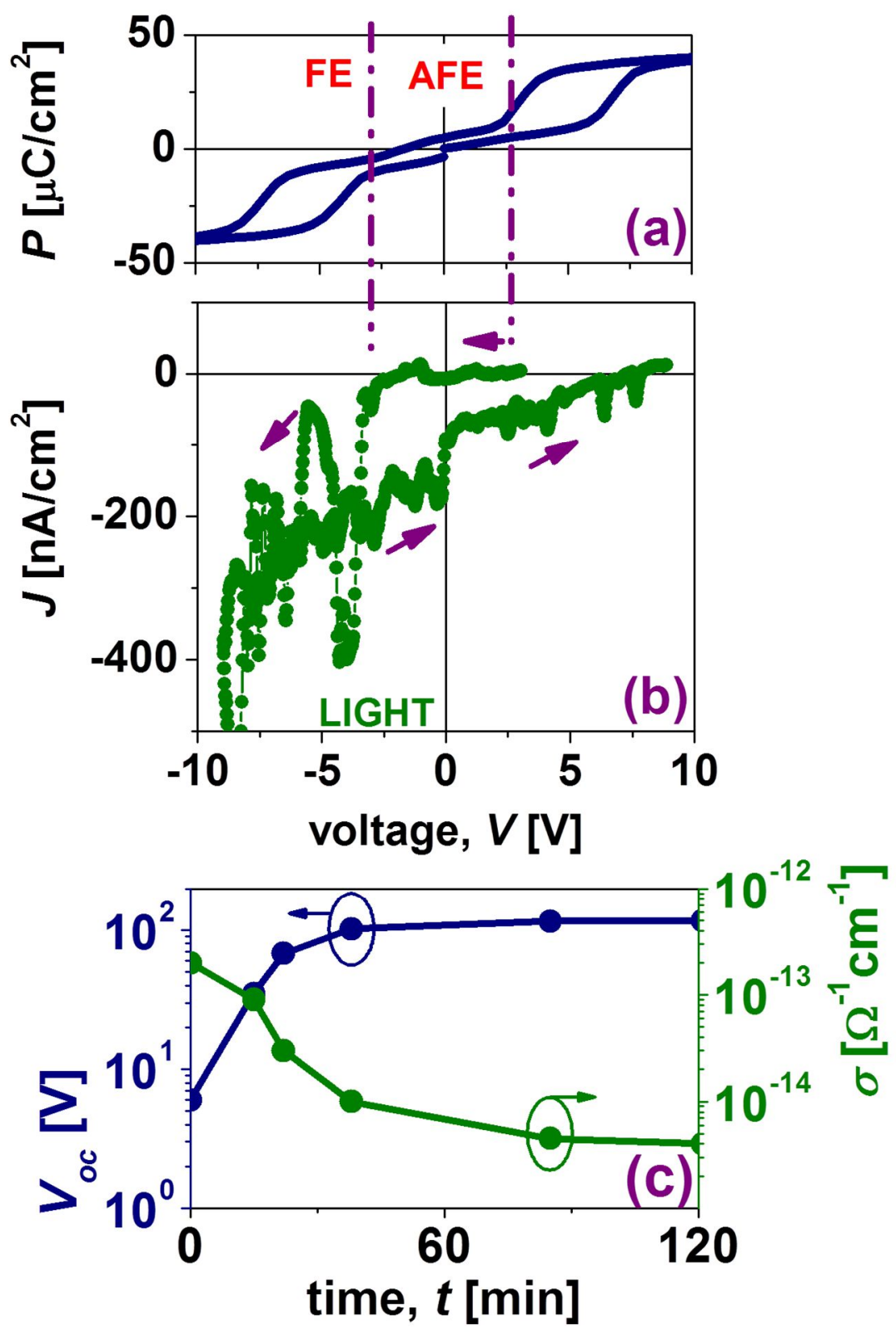

Fig. 2. (a) Antiferroelectric hysteresis loop measured in dark and (b) current $v s$ voltage response under illumination of an antiferroelectric thin film capacitor. The virgin antipolar phase shows no photocurrent, but when the film switches from the antipolar to the polar phase, a displacement current peak appears, after which a steady-state photocurrent is established and remains even when the external bias is switched off (short-circuit photocurrent). The induced polar state displays above-bandgap photovoltage. (c) time evolution of the photoconductivity and open-circuit photovoltage. 

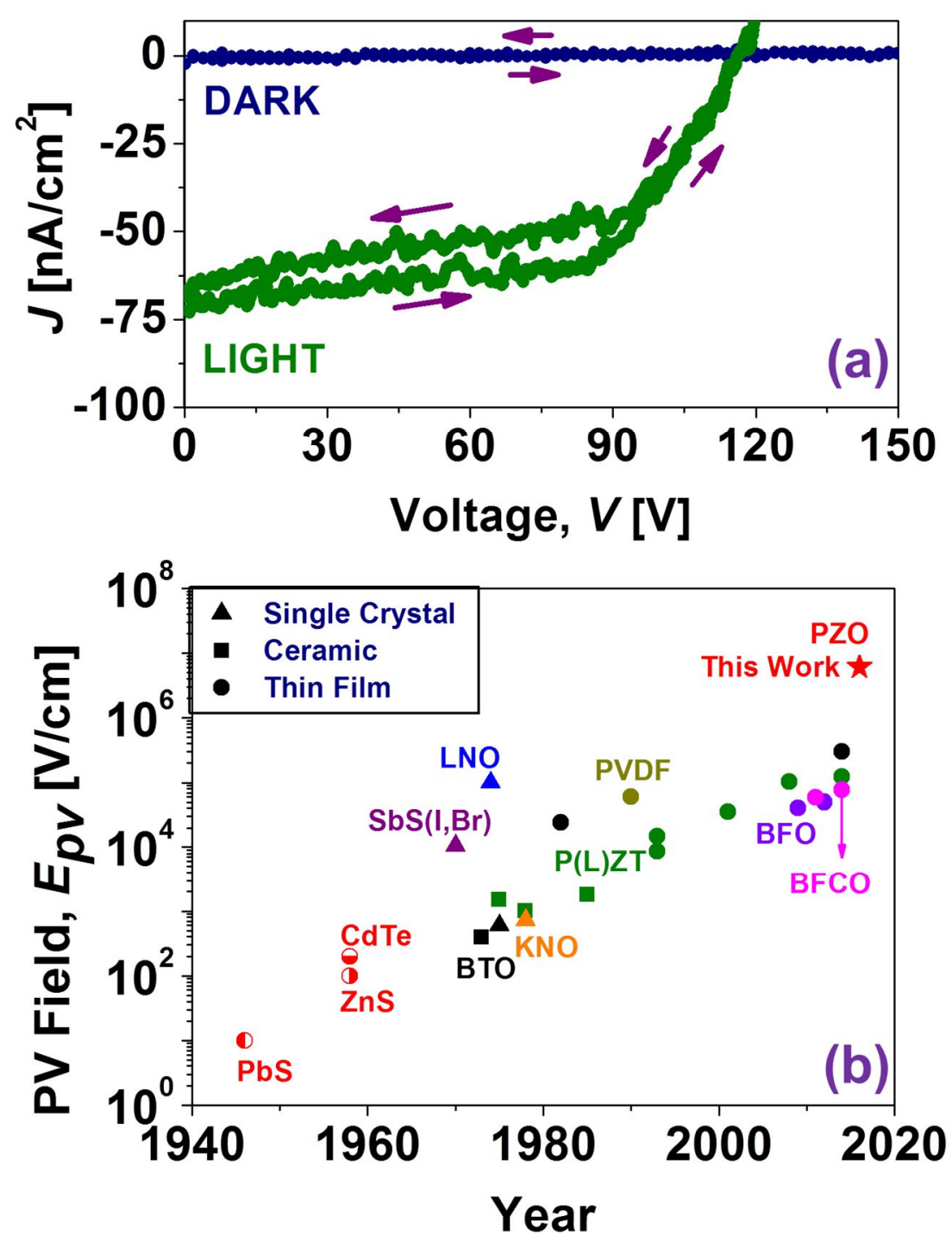

Fig. 3. (a) steady-state photovoltaic response of the capacitor, showing an open-circuit photovoltage in excess of 100V. (b) A timeline review (references are listed under Supplementary section III) of the reported photovoltaic fields for different above bangap and/or ferroelectric photovoltaic materials from the literature. Non-polar piezoelectrics (CdTe, $\mathrm{ZnS}, \mathrm{PbS}$ ) stay below the $\sim \mathrm{kV} / \mathrm{cm}$ range, which is only achieved by ferroelectric materials such as $\mathrm{BTO}\left(\mathrm{BaTiO}_{3}\right), \mathrm{KNO}\left(\mathrm{KNbO}_{3}\right), \mathrm{LNO}\left(\mathrm{LiNbO}_{3}\right), \mathrm{BFO}\left(\mathrm{BiFeO}_{3}\right)$ and $\mathrm{BFCO}$ $\left(\mathrm{Bi}_{2} \mathrm{FeCrO}_{6}\right)$. PVDF is polyvinylidene fluoride, a ferroelectric polymer. The antiferroelectric PZO photovoltaic cells are the first to reach the $\mathrm{MV} / \mathrm{cm}$ range. 